\title{
Diamante Digital, una apuesta territorial por el desarrollo a partir de las TIC
}

\section{Diamante Digital, a territorial commitment to development based on ICT}

\author{
Pedro M. Wightman ${ }^{1}$ (D), Wilson Nieto ${ }^{1}$ (D), Jaider Vega ${ }^{3}$ (D), Miguel Jimeno ${ }^{1}$ (D), Lucy \\ García $^{1}$ (D), Mauricio Pardo ${ }^{2}$ (D), Diego Gómez ${ }^{2}$, Emyle Britton $^{3}$ (D), Andrea Pérez ${ }^{1}$ \\ ${ }^{1}$ Departamento de Ingeniería de Sistemas, Universidad del Norte, Barranquilla, Colombia \\ ${ }^{2}$ Departamento de Ingeniería Eléctrica y Electrónica, Universidad del Norte, Barranquilla, Colombia \\ ${ }^{3}$ Departamento de Finanzas y Organizaciones, Universidad del Norte, Barranquilla, Colombia \\ \{pwightman, wnieto, jaiderv, majimeno, lucyr, mpardo, dgomez, ebritton, areza\}@uninorte.edu.co
}

(Recibido: 22 agosto 2018; aceptado: 4 diciembre 2018)

\begin{abstract}
Between January 2014 and September 2015, the Diamante Caribe and Santanderes initiative was carried out: a joint territorial planning exercise between the nine departments that make up the Colombian regions of the north coast of the country and the santanderes of Colombia. The result of this project was the definition of an integrated vision of growth, based on a sectoral diagnosis, which included a portfolio of projects with different impacts on the region. One of the sectors selected for this study was Technological Platforms, which includes information systems and IT platforms, city instrumentation, electronic government, health, education, industrial instrumentation. This work summarizes the experience of the development of this study, and the final vision of a regional strategy for ICTs called Digital Diamond. In addition, some complementary results and lessons learned from the process are presented.
\end{abstract}

Keywords: ICTs; Urban development; Territorial planning; Local government; Information systems

\section{Resumen}

Entre enero de 2014 y septiembre de 2015, se llevó a cabo la iniciativa Diamante Caribe y Santanderes: un ejercicio de planeación territorial conjunta entre los nueve departamentos que conforman las regiones colombianas de la costa norte del país y los santanderes. El resultado de este proyecto fue la definición de una visión integrada de crecimiento a partir de un diagnóstico sectorial, que comprende un portafolio de proyectos con impactos de diferente índole sobre la región. Uno de los sectores seleccionados para este estudio fue el de Plataformas Tecnológicas, el cual comprende sistemas de información y plataformas TI, instrumentación de ciudad, gobierno electrónico, salud, educación, instrumentación industrial. Este trabajo resume la experiencia del desarrollo de este estudio, y la visión final de una estrategia regional para las TIC denominada Diamante Digital. Además, se presentan algunos resultados complementarios y lecciones aprendidas del proceso.

Palabras clave: TIC; desarrollo urbano; planeación territorial; gobierno local; sistemas de información; 


\section{Introducción}

El Siglo XX ha sido testigo de un proceso acelerado de urbanización de los grupos humanos: de acuerdo con la División de Asuntos Económicos y Sociales de la ONU, de un 30\% de la población mundial que vivió en ciudades durante 1950 , se pasó a un 54\% en el 2014, y se proyecta que esta cifra seguirá subiendo hasta un 66\% en el año 2050 (Department of Economic and Social Affairs, 2018). Sin embargo, en el mismo informe se observa que América Latina tiene un $80 \%$ de población viviendo en centros urbanos. Este hecho representa tanto una problemática como una oportunidad. Las ciudades se han convertido en el destino principal para buscar oportunidades de desarrollo para la población: trabajo, educación, salud, servicios públicos, entre otros. Las ciudades ya no son vistas desde una óptica individualista o unitaria en la economía, por el contrario, son concebidas como un punto estratégico en el que se gestan y fortalecen las ventajas competitivas de los países, pues es en ellas donde se concentra la capacidad de innovación tecnológica y empresarial, el talento con conocimiento y el capital necesario para lograr la competitividad territorial (Komninos, 2002). Pero, al mismo tiempo, con una población creciente, la planeación de las ciudades debe hacerse de manera exhaustiva, pues los recursos para invertir en el aseguramiento de los servicios para la población son finitos.

Asimismo, las nuevas tecnologías se han convertido en una herramienta fundamental no solo para acceder y transmitir información, sino también para crear redes de conexión que permiten la interacción entre toda la población mundial a través del intercambio de conocimientos, culturas e ideologías. No obstante, estas tecnologías han dado paso al surgimiento de una brecha digital, construida por la dificultad que tiene un gran porcentaje de la población para acceder a las herramientas del mundo digital, especialmente por sus altos costos de adquisición y por el nivel de educación y cualificación que implica su uso.

Los hechos antes mencionados han propiciado que los modelos tradicionales de gestión de los territorios se conviertan en ineficientes y no respondan de manera adecuada a los desafíos que propone el mundo económico, social y político contemporáneo; en otras palabras, han impulsado la necesidad de proponer nuevos patrones de desarrollo que conduzcan a una renovación de la economía, de las formas de gobernar el territorio, de la infraestructura física y que tengan como principal eje transversal el desarrollo e implementación de las tecnologías de la información y comunicación y la aplicabilidad del concepto de sostenibilidad no solo desde el punto de vista ambiental, sino también social y económico (Rogers \& Gumuchdjian, 1997).

Es precisamente en el marco de la búsqueda de nuevos modelos de desarrollo que ha emergido en los últimos años la noción de Territorio Inteligente, para caracterizar aquellos espacios que son capaces de encontrar un equilibrio entre los aspectos clave para el buen funcionamiento de los territorios, respondiendo adecuadamente a los retos de sostenibilidad y competitividad. Teniendo en cuenta las tendencias y desafíos actuales, este enfoque se muestra como un marco de referencia adecuado para el diseño de estrategias de desarrollo en la medida en que parte del reconocimiento del propio territorio y de sus potencialidades como base para afrontar los retos de una economía cada vez más globalizada, al tiempo que integra la ciencia, la tecnología, y la innovación como los pilares del desarrollo en una sociedad basada en el conocimiento.

Cuando se habla de territorio no necesariamente se hace referencia a una ciudad; de hecho, a la creciente importancia de las ciudades como unidades de desarrollo competitivo se suma una nueva escala de análisis: la región. En las zonas más dinámicas del mundo, la expansión de las ciudades está dando lugar a procesos de integración a escala regional que han demostrado tener un gran potencial para fomentar el crecimiento y el desarrollo sostenible de un país. Estas nuevas organizaciones territoriales, denominadas Diamantes Urbanos o superciudades, se están convirtiendo en los nuevos motores del crecimiento económico a nivel mundial (Fundación Metrópoli, 2013). Sin embargo; en Colombia, este tipo de procesos no ha tenido lugar y, de hecho, la segmentación del mercado nacional y los escasos esfuerzos de integración de las ciudades pueden señalarse como algunos de los factores que han contribuido a que el país haya avanzado poco en materia de competitividad.

La región Caribe y los Santanderes constituyen un espacio que tiene grandes potencialidades para llegar a convertirse en uno de estos territorios, catalogados como diamantes urbanos. Su localización estratégica, sus condiciones demográficas y la diversidad de sus recursos ambientales, sociales, culturales y económicos, ofrecen amplias posibilidades para impulsar espacios innovadores, sostenibles y competitivos. Desde el punto de vista de su localización geográfica, la integración de los departamentos de la región Caribe y Santanderes conformaría un espacio clave tanto para el comercio exterior colombiano como para la articulación de flujos logísticos que aumenten la competitividad de la economía nacional. 
El proyecto Diamante Caribe y Santanderes surgió en el marco antes expuesto y tuvo como objetivo analizar los factores clave de competitividad de la región Caribe y Santanderes para el diseño de un modelo de desarrollo territorial y urbano inspirado en el concepto de Territorio Inteligente. Este Proyecto, financiado con recursos de los departamentos de la región Caribe y Santanderes provenientes del fondo de Ciencia, Tecnología e Innovación del sistema General de Regalías, contemplaba dos grandes fases. La primera estaba orientada a la realización de un proceso de investigación territorial orientada a la identificación de los componentes de excelencia del territorio y la definición de un modelo de Territorio Inteligente para la región. La segunda fase consistía en la operacionalización de dicho modelo a través de la formulación de proyectos estratégicos que contribuyeran a la transformación del territorio.

Un aspecto clave para el diseño, la implementación y puesta en marcha de estrategias de desarrollo de territorios inteligentes y sostenibles es, sin lugar a duda, el desarrollo y apropiación de las TIC por parte de los diferentes actores. Las TIC constituyen un eje estratégico que apoyan el despliegue de iniciativas en los demás componentes de un territorio, a saber: economía, población, gestión de las ciudades e industrias, salud, educación, gobierno y ciudadanía y, por último, medio ambiente. Los territorios que logran estadios de inteligencia son aquellos que utilizan estratégicamente las TIC para integrar sus subsistemas críticos, promoviendo a su vez el desarrollo sostenible y la innovación. En este sentido, el diseño de estrategias que fomenten la transformación de una región en un territorio inteligente se debe considerar como elemento transversal.

Este artículo presenta parte de los resultados del Proyecto señalado anteriormente, relacionados con el análisis del componente tecnológico. Para ello, se inicia con una revisión del enfoque de territorio inteligente, especialmente desde su dimensión tecnológica. Posteriormente se describe la metodología utilizada en el estudio para caracterizar la infraestructura digital existente en el territorio, así como para definir un banco de proyectos que promuevan la consolidación del componente digital del territorio; y finalmente, se presentan los resultados.

\section{Territorios Inteligentes y las TIC}

El concepto de Territorio Inteligente es muy reciente, por lo que aún no existe en la literatura una definición precisa y consensuada de Smart City. Una posible explicación sobre la falta de un consenso en la literatura puede explicarse a la luz de la visión que presentan Neirotti et al., quienes opinan que este término ha sido empleado de una manera demasiado amplia, tanto en contextos técnicos como en contextos generales, sin poder abarcar el amplio espectro de subáreas que esta comprende y su complejidad inherente (Neirotti, De Marco, Corinna Cagliano, Mangano, \& Scorrano, 2014).

Las primeras ideas que contribuyen al concepto de territorios inteligentes vienen del punto de vista urbanístico y de la institucionalidad, incluyendo el "Smart Growth Movement" el cual, a finales de los años 90 del siglo XX, perseguía el desarrollo de nuevas políticas de planificación y desarrollo urbano (Harrison \& Donelly, 2011). Por su parte, Albino Berardi, \& Dangelico (2015) opinan que el término fue introducido con el objetivo de resaltar la implementación de las nuevas tecnologías en las infraestructuras de las ciudades . Esta iniciativa también fue adoptada por el California Institute for Smart Communities, que fue uno de los primeros movimientos en investigar cómo las comunidades podían adquirir "inteligencia" y cómo las ciudades podían implementar las TIC en sus procesos (Alawadhi et al., 2012).

Siguiendo esta línea de pensamiento, se puede rescatar la idea de "Ciudad Digital" o "Ciudad Cableada": un espacio pensado para el intercambio de información entre los diferentes actores, posible gracias a una infraestructura de comunicaciones y computación adecuada, accesible para todos los miembros de la comunidad (sociedad, industria, gobierno, academia), con el fin de asegurar que estén interconectados (Dutton, Blumler, \& Kraemer, 1987; Ishida \& Isbister, 2000; Yovanof \& Hazapis, 2009). Otro concepto relacionado es el de "Ciudad Ubicua" en donde la interconexión entre los actores no es suficiente sino que existe una infraestructura de monitoreo constante y de diferentes variables, desde ambientales hasta comportamentales de la comunidad, que permite conocer el estado de la ciudad y sus habitantes en cualquier momento (Hyang-Sook, Byung-Sun, \& Woong-Hee, 2007; Leem, Lee, \& Yoon, 2014). Por último, el concepto de Ciudad Híbrida (Streitz, 2011) incluye una visión de ciudad donde los espacios físicos habitados por ciudadanos convergen con una ciudad digital disponible gracias a las TIC.

En los últimos años, el concepto de "inteligente" ha migrado hacia algo más allá que solo la implementación de la tecnología y el acceso a ella, pues esto no necesariamente garantiza su uso para los procesos urbanos. En la actualidad, la inteligencia de la ciudad se asocia más con la capacidad de producir y procesar información en tiempo real obtenido a través de una infraestructura formal de instrumentación 
urbana (sensores, cámaras, etc.), medios de comunicación emergente (redes sociales, blogs, aplicaciones móviles) e incluso información generada por privados (compañías de transporte público, operadores de telecomunicaciones, etc.). La posibilidad de generar, procesar, usar y comunicar los datos de fuentes tan distintas para generar una visión unificada de ciudad entre todos los actores, y poder planificar el desarrollo urbano a mediano y largo plazo de manera conjunta, apoyando la definición de política pública basada en hechos, demuestra el gran potencial sin precedentes que proveen las TIC en la actualidad (Marsal-Llacuna, Colomer-Llinàs, \& Meléndez-Frigola, 2015; Yin et al., 2015; Zygiaris, 2013).

Por lo tanto, y teniendo en cuenta los conceptos anteriores, un Territorio Inteligente debe contar con una infraestructura tecnológica base que permita a los habitantes, sean estos individuos, empresas o el mismo gobierno, interactuar, compartir datos, experiencias y opiniones, para luego usar todos estos datos y planear de manera conjunta, participativa, transparente e informada, el desarrollo urbano del espacio compartido por todos. El resultado del trabajo hecho en la región incluye una etapa de diagnóstico de esta infraestructura, la identificación de fortalezas, y aspectos por mejorar de estos recursos y un conjunto de propuestas para potencializar las vocaciones intrínsecas de los diferentes departamentos, que se denomina el Diamante Digital.

\section{Metodología}

Este estudio puede ser catalogado, principalmente, como exploratorio, aunque con componentes de una investigación-acción, puesto que no solo indaga la situación actual de los departamentos en cuanto al estado de infraestructura tecnológica, sino que propone áreas y proyectos de intervención, con participación de los actores interesados. Este estudio se definió en tres (3) grandes fases: 1) diagnóstico del componente tecnológico en la región, el cual fue realizado a través de fuentes de información primaria y secundaria, 2) formulación de perfiles de proyectos con base en las fortalezas y debilidades identificadas en la región, 3) validación por parte de los stakeholders sobre el diagnóstico y los perfiles de proyectos, y 4) formulación del banco de proyectos para el Diamante Digital.

\section{Diagnóstico del componente TIC}

El proceso de diagnóstico buscaba hacer un levantamiento de información primaria y secundaria sobre el estado actual de las plataformas tecnológicas, con el fin de identificar los aspectos a mejorar y las potencialidades del territorio.

La información de fuentes secundarias fue tomada principalmente de cifras oficiales provistas por el Ministerio de las Tecnologías de la Información y las Comunicaciones - MinTIC, el Departamento Administrativo Nacional de Estadística - DANE, Comisión de Regulación de Comunicaciones - CRC, Dirección Nacional de Planeación - DNP, entre otros entes del ámbito regional, nacional e internacional. Las cifras aglutinan el esfuerzo nacional para la recolección de datos tradicionales como penetración tecnológica en la población, infraestructura instalada, entre otros.

Sin embargo, a pesar de la utilidad de esa información que permite conocer el estado de la infraestructura base para la creación de un territorio inteligente, es necesario también medir el tipo de uso que esta infraestructura tiene, las capacidades de los agentes del gobierno para acceder y operar tecnologías emergentes, entre otros temas que no son cubiertos por las encuestas tradicionales; además, se definieron seis (6) áreas al interior del proyecto de alto interés y gran potencial de uso de las TIC en pro del desarrollo regional: Plataforma TI, Salud, E-Gobierno, Educación, Instrumentación industrial, e Instrumentación de ciudad. Por esta razón, dentro del proyecto se diseñaron instrumentos que serían aplicados a los entes territoriales para que les permitiera plasmar su percepción.

\subsection{Formulación de perfiles de proyectos}

A partir de la información recogida sobre las áreas de estudio, tanto de fuentes primarias como secundarias, se pasó a realizar un ejercicio FODA para identificar las fortalezas, oportunidades, debilidades y amenazas en el territorio respecto de su estado actual en infraestructura y uso de las TIC. Esta información luego sirvió de base para la formulación de perfiles de proyectos puntuales que buscan potencializar fortalezas, concretar oportunidades, apoyar la solución de debilidades o mitigar las amenazas a largo plazo.

La escala de implementación de los perfiles varía entre soluciones generales para todo el territorio hasta soluciones muy puntuales para los centros urbanos. Estos perfiles debían incluir una definición del problema, justificación, objetivos, metodología y aspectos muy generales, mas no aspectos específicos 
como presupuesto o cronograma. Esta decisión se tomó debido a que el alcance de tiempo del proyecto no alcanzaba para una definición detallada de los mismos y porque antes debían ser validados por los actores.

En esta fase se definieron 17 perfiles de proyecto que apuntaban a todas las áreas de estudio y que buscaban sentar las bases de infraestructura de apoyo para la realización de los grandes perfiles de proyecto a nivel de ciudad y de territorio propuestos en el proyecto por Fundación Metrópoli y Microsoft.

\subsection{Validación con stakeholders}

Una vez los proyectos fueron definidos y la información primaria fue tabulada, se procedió a convocar una sesión de trabajo con actores que representaban industria, academia, gobierno local y gobierno nacional para que retroalimentaran los resultados y los perfiles de proyectos, especialmente a nivel de orientación, pertinencia y alcance. Además, cada uno de los asistentes pudo compartir iniciativas existentes en sus departamentos que complementaban las propuestas presentadas, o dar sugerencias sobre aspectos por mejorar.

Además, se llevó a cabo una segunda medición de indicadores con una nueva versión de los instrumentos de diagnóstico con el fin de validar la información de fuentes primarias previamente obtenidas, teniendo en cuenta sugerencias sobre la extensión y complejidad de las encuestas iniciales.

\subsection{Banco de proyectos del Diamante Digital}

Finalmente, una vez terminado el proceso de validación de los perfiles, se procedió a generar un segundo ejercicio de formulación de proyectos con un mayor nivel de detalle, que apoyaran la conformación de una iniciativa de mayor envergadura para la región, potencializando los perfiles más asertivos en cuanto a las necesidades consultadas con los actores, y que extendieran su alcance más allá de los centros urbanos, por ejemplo, en temas relacionados con agro y áreas rurales.

Este nuevo ejercicio generó nueve (9) proyectos que decantan las necesidades de la región en temas relacionados con sus plataformas tecnológicas en relación con sus fortalezas y oportunidades. Esta formulación incluye aspectos como posibles actores y fuentes de financiación, y presupuesto tentativo, con lo que se espera que los proyectos puedan ser presentados a los entes territoriales para ser madurados como proyectos completos y presentados a convocatorias nacionales e internacionales, fondos del Sistemas General de Regalías, entidades financieras o entes multilaterales para obtener fondos y ejecutar.

\subsection{Participantes}

En la primera fase, dada la naturaleza piloto de los instrumentos, se consideró una muestra inicial del paquete de encuestas por ciudad, diligenciada por el secretario de informática o similar, con el apoyo de los secretarios de salud, educación y planeación para profundizar en información pertinente y muy particular para ciertos indicadores; por ejemplo, servicios de telemedicina, infraestructura de cómputo y software disponible en los colegios, sensores ambientales en la ciudad, entre otros.

En la fase de validación con stakeholders se extendió una invitación a miembros de los gobiernos locales, tanto a nivel departamental como de alcaldías de las ciudades capitales, académicos, miembros de la industria local y representantes del Ministerio TIC. Se contó con la asistencia de 12 representantes de estos sectores, incluyendo gremios como CaribeTIC, el clúster de empresas TIC de la costa caribe, el Viceministerio TIC, entre otros.

\section{Instrumentos}

\subsection{Encuestas para diagnóstico}

Para cada una de las áreas de estudio se procedió a identificar indicadores relevantes, tratando de evitar repetir aquellos definidos en los reportes consultados en la fase de diagnóstico. El principal objetivo era hacer un levantamiento de aspectos relacionados con el uso de las plataformas y la infraestructura que no se ve reflejada en los estudios estandarizados. A continuación, se presentan los aspectos generales que fueron evaluados en cada uno de los instrumentos. 


\section{Plataforma de TI}

La estructura de este instrumento está basada en los siguientes aspectos: penetración de infraestructura de telecomunicación y de tecnología informática, infraestructura de tecnología informática, infraestructura de apoyo, manejo de información, y nivel de interconexión y colaboración.

En la sección de penetración de infraestructura de telecomunicación y de tecnología informática se busca establecer el nivel de adopción de servicios como telefonía fija, telefonía móvil, internet fijo, internet móvil, redes de transmisión a nivel domiciliario, empresarial y gubernamental. Se pide valorar si el nivel de la infraestructura existente, el nivel de cobertura, el papel de los actores, y el costo del servicio permiten la prestación de servicios de telecomunicación de una forma adecuada. Esta sección cubre algunos aspectos que se evalúan en reportes oficiales, pero se usó como elemento de validación de resultados.

En la sección de infraestructura de tecnología informática se busca establecer si el nivel de conocimiento, experiencia, y uso de las tecnologías de cómputo en entes gubernamentales es el adecuado para implementar soluciones. Se pide valorar si los entes gubernamentales conocen y usan infraestructura de tecnologías informáticas en sus actividades, procesos y servicios. Además, se pide valorar si los entes gubernamentales conocen, tienen experiencia y usan tecnologías avanzadas en sus actividades, procesos y servicios, que incluyen Big Data, aplicaciones móviles, aplicaciones web, entre otros.

En la sección de infraestructura de apoyo se busca establecer qué entidades son consideradas como parte de la infraestructura de apoyo para la implementación de infraestructura de telecomunicaciones. Se pide valorar el nivel de impacto actual y deseado de los siguientes actores para el diseño, implementación y mantenimiento de la infraestructura de telecomunicaciones y de infraestructura informática. Entre estos actores se incluyen la academia; los gremios; la industria, a escala local, nacional e internacional.

En la sección de gestión de la información se busca establecer qué tan bien están preparados los entes gubernamentales para administrar la información que tienen sobre la ciudad. Se pide valorar el nivel de conocimiento, experiencia, planes de uso, y uso actual de las fases de administración de la información de la ciudad. Este elemento busca definir el nivel de madurez en la capacidad de análisis de datos de la ciudad en los ejercicios de planeación de la misma.

Finalmente, en la sección de nivel de interconexión y colaboración se busca establecer qué tan bien preparados están los entes gubernamentales para trabajar de manera conjunta. Se pide valorar el nivel de comunicación, interconexión y/o colaboración con los siguientes actores, haciendo uso de las tecnologías de la información y comunicación.

\section{E-Government}

Varias son las referencias encontradas para la definición de Gobierno Electrónico o e-government; en principio cabe mencionar (Deloitte Research, 2000; Division for Public Institutions and Digital Government, 2018; Grima-Izquierdo, 2010; Hai \& Jeong, 2007; Kaylor, Deshazo, \& Van Eck, 2001), a quienes coinciden ampliamente en definir el gobierno electrónico e-government -abreviatura para gobierno electrónico, gobierno digital, gobierno en línea, o el gobierno conectado- como el espacio de las interacciones digitales entre un ciudadano y su gobierno $(\mathrm{C} 2 \mathrm{G})$, entre los gobiernos y las agencias gubernamentales $(\mathrm{G} 2 \mathrm{G})$, entre el gobierno y los ciudadanos (G2C), entre el gobierno y los empleados (G2E), y entre el gobierno y los negocios / comercio (G2B). Esencialmente, los modelos de prestación de gobierno electrónico se pueden resumir brevemente como Hai \& Jeong (2007) las presentan: G2G (government to governments), G2C (government to citizens), G2E (government to employees), G2B (government to businesses), cuya interacción digital consiste en la participación del e-ciudadano en todos los niveles de gobierno (ciudad, estado / provincia, nacional e internacional), para acceder a la gobernanza, la información y a las tecnologías de la información y la comunicación (TIC), y el proceso de reingeniería de negocios (BPR) de los estados para el mejoramiento de su eficiencia y la prestación de servicios.

La estructura de instrumento está dividida en las siguientes secciones: entrega de servicios on line, transparencia, open government data, e-participación, y formación del ciudadano digital.

En la sección de entrega de servicios on line se busca identificar el uso de las TIC por parte de los entes gubernamentales (Gobernación, Alcaldía, Institutos u otros) para entregar servicios públicos on line a los ciudadanos. Se pide valorar la disponibilidad de servicios a los ciudadanos como transacciones, quejas y reclamos, foros de participación ciudadana, mapas interactivos, entre otros. 
En la sección de transparencia se busca identificar las plataformas digitales existentes que promueven la transparencia en la gestión pública, en materia de divulgación de información de interés público desde los entes gubernamentales (Gobernación, Alcaldía, Institutos u otros). Se pide valorar el nivel de accesibilidad de información a labores como ejecución presupuestal, contratación, planes de gobierno, entre otros.

En la sección de gobierno abierto se busca identificar cuáles son las herramientas que utilizan los entes gubernamentales para aumentar la transparencia y la participación bajo la estrategia de datos abiertos gubernamentales u Open Government Data - OGD, la cual puede definirse como la filosofía que promueve transparencia, responsabilidad, y creación de valor a partir de hacer públicos los datos gubernamentales, su uso y su redistribución gratuita, con el fin de impulsar la creación de servicios centrados en el ciudadano, con modelos de negocios innovadores, por parte de la misma comunidad (OCDE, 2018). Se busca evaluar si el gobierno local cuenta con datasets disponibles para consumo por parte de actores ciudadanos.

En la sección de e-participación se busca identificar si los entes gubernamentales disponen de plataformas digitales que faciliten acceder a los ciudadanos a participar en los mecanismos de participación ciudadana; por ejemplo: las iniciativas populares legislativas y normativas, el referendo, la consulta popular de orden nacional, departamental, distrital, municipal y local, la revocatoria del mandato, el plebiscito y el cabildo abierto. Además, se explora la disponibilidad de accesibilidad a recursos informáticos, tanto a nivel de software como de recursos físicos para poder llevar a cabo la participación: ventanilla única, plataformas móviles, redes sociales, quioscos públicos, etc.

Finalmente, en la sección de formación del ciudadano digital se busca conocer cuáles son las acciones del Estado encaminadas a desarrollar programas de alfabetización digital con el objeto de masificar las competencias del ciudadano en el manejo de la información y los recursos TIC, incluyendo programas de formación en TIC para la población ciudadana general, población vulnerable, contenido en lenguas nativas, entre otros.

\section{Salud}

Este instrumento está dividido en las siguientes secciones: prestación de servicios primarios de salud, cuidado remoto y manejo de casos, salud personal y bienestar, manejo de pandemias y emergencias sanitarias, y manejo de información sobre jubilados.

En la sección de prestación de servicios primarios de salud se busca establecer el nivel de penetración de servicios TIC en la prestación de estos, que consisten en la asistencia sanitaria esencial accesible a todos los individuos y familias de la comunidad a través de medios aceptables para ellos, con su plena participación y a un costo asequible para la comunidad. Se pide valorar si los entes gubernamentales (Gobernación, Alcaldía, Institutos u otros) ofrecen herramientas digitales necesarias para garantizar una adecuada prestación de servicios primarios de salud.

En la sección de cuidado remoto y manejo de casos, se busca establecer el nivel de preparación del sistema de salud para atender remotamente casos médicos que no necesiten atenderse en una clínica o en el consultorio de un especialista. Se pide valorar si los entes gubernamentales ofrecen herramientas digitales necesarias para ofrecer atención médica remota.

En la sección de salud personal y bienestar se busca establecer qué tantas herramientas y de qué calidad tienen los entes gubernamentales para enfrentar problemas de salud pública como obesidad, abuso del alcohol, diabetes, enfermedades cardiovasculares, hipertensión y estrés. Se pide valorar las herramientas digitales -ofrecidas actualmente para apoyar los procesos de prevención, seguimiento y atención- que el ciudadano puede utilizar por sí mismo para complementar la atención médica.

La sección de manejo de pandemias y emergencias sanitarias pretende establecer qué tan bien están preparados los entes gubernamentales para atender urgencias de pandemias; por ejemplo, enfermedades estacionales con picos de transmisión como la influenza, o casos puntuales de insalubridad causadas por alimentos mal manejados. Se pide valorar si los entes gubernamentales ofrecen herramientas digitales necesarias para atender este tipo de eventos.

Por último, en la sección de manejo de información sobre jubilados se busca establecer el nivel de preparación en que se encuentra el sistema de salud de la región para manejar la información sobre jubilados y personas de la tercera edad en general, para poder brindarle asistencia en sus necesidades de manera adecuada y oportuna. Se pide valorar las plataformas existentes que permitan la gestión de la información, seguimiento y gestión de asuntos relacionados con la población de la tercera edad y jubilados. 


\section{Educación}

El instrumento de educación está dividido en las siguientes secciones: infraestructura TIC, recursos educativos digitales, competencias TIC, y planificación y gestión TIC.

La sección de infraestructura TIC pretende medir el estado actual de la infraestructura de TIC que permite el acceso a servicios digitales educativos. Se le pide a los consultados valorar si los entes gubernamentales (Gobernación, Alcaldía, Institutos u otros) ofrecen los recursos tecnológicos que le permiten a las instituciones educativas acceder a la oferta de productos y servicios educativos.

La sección de recursos educativos digitales busca medir el estado actual de los recursos educativos digitales que facilitan los procesos de enseñanza-aprendizaje en la educación preescolar, básica y secundaria. Se pide valorar si los entes gubernamentales (Gobernación, Alcaldía, Institutos u otros) ofrecen los recursos educativos digitales que facilitan los procesos de enseñanza-aprendizaje, como software educativo, software para la gestión del aprendizaje, sistema de seguridad digital para contenidos inapropiados, entre otros.

En la sección de competencias TIC se pretende medir el nivel de apropiación de las TIC para facilitar los procesos de enseñanza-aprendizaje en la educación preescolar, básica y secundaria. Se pide valorar si los entes gubernamentales (Gobernación, Alcaldía, Institutos u otros) promueven la apropiación de las competencias TIC para facilitar los procesos de enseñanza-aprendizaje, como la integración de TIC en los procesos educativos, posibilidad de crear redes o comunidades académicas, entre otros.

Finalmente, la sección de planificación y gestión TIC busca medir el nivel de apropiación de las TIC para facilitar los procesos de planificación y gestión de las TIC para facilitar los procesos de enseñanzaaprendizaje en la educación preescolar, básica y secundaria. Se le pide al evaluador que valore si los entes gubernamentales (Gobernación, Alcaldía, Institutos u otros) promueven el desarrollo de estrategias, políticas o planes de acción para fomentar el uso de las TIC para mejorar la calidad de los procesos de enseñanza-aprendizaje en la educación preescolar, básica y secundaria.

\section{Instrumentación de ciudad}

La estructura de este instrumento está dividida en las siguientes secciones: movilidad, seguridad, clima y medio ambiente, y servicios públicos.

En la sección de movilidad se busca identificar los servicios disponibles para la ciudad y así apoyar la planeación, el seguimiento y la administración de la movilidad en la ciudad. Se pide valorar el uso de plataformas tecnológicas en distintos ámbitos de la movilidad que permiten un mejor sistema de tránsito y un mejor uso del transporte público; entre ellas se incluyen: monitoreo de rutas y control de tiempo, taxímetro y control de precio, cámaras y foto-multas, entre otros.

En la sección de seguridad se busca identificar el nivel de implementación de infraestructura para apoyar el monitoreo de la seguridad en la ciudad. Se pide valorar el uso de plataformas tecnológicas como cámaras públicas, aplicaciones móviles para reporte de incidentes, sistemas de georreferenciación para optimizar tiempo de respuesta, entre otros.

En la sección de clima y medio ambiente se busca identificar la infraestructura disponible para el monitoreo y seguimiento de variables climáticas y ambientales de la región. Se pide valorar el uso de plataformas tecnológicas como estaciones de monitoreo, pronóstico y sistemas de alerta temprana de eventos y catástrofes, disponibilidad de plataformas de consulta para los ciudadanos, entre otros.

Finalmente, en la sección de servicios públicos se busca identificar el uso de plataformas tecnológicas destinadas a complementar la prestación de los servicios públicos para una mejor experiencia del usuario. Se incluyen plataformas como servicios en línea, posibilidad de generar quejas y reclamos, sistema de medición, entre otros.

\section{Instrumentación industrial}

El instrumento de instrumentación industrial incluye las siguientes secciones: infraestructura tecnológica a nivel de planta, implementación de sistemas de información para toma de decisiones gerenciales (ERP), infraestructura tecnológica para la interacción entre planta y la estructura administrativa, y herramientas tecnológicas para comunicación con clientes y proveedores.

En la sección de infraestructura tecnológica a nivel de planta se busca establecer el nivel de acceso a soluciones de automatización industrial para optimización de procesos productivos por parte de las empresas del sector industrial colombiano, tanto a nivel de infraestructura instalada como a nivel de formación del 
personal en su uso. Se incluyen tecnologías como sistemas supervisores de planta (SCADA), plataformas para el control y automatización de procesos (PLCs, PAC), entre otras.

En la sección de implementación de sistemas de información para toma de decisiones gerenciales (ERP), se pretende medir el nivel de acceso a sistemas de información para toma de decisiones gerenciales por parte de las empresas del sector productivo colombiano. Se pide valorar si las empresas del sector industrial colombiano implementan sistemas de información como respaldo a procesos como: gestión de materia prima, monitoreo de variables de planta, gestión de inventario, entre otros.

La sección de infraestructura tecnológica para la interacción entre planta y la estructura administrativa busca establecer el nivel de importancia que se le asigna a la implementación de un sistema de información que permita la interacción entre los procesos llevados a cabo en planta (misionales) y los procesos a nivel administrativo (de soporte). Se pide valorar qué tan importante resulta la implementación de herramientas tecnológicas que permiten la interacción entre los procesos administrativos y productivos de la planta.

Finalmente, la sección de herramientas tecnológicas para comunicación con clientes y proveedores permite identificar la infraestructura tecnológica para la comunicación de la empresa con sus clientes y proveedores. Se le pide al encuestado que valore las plataformas tecnológicas utilizadas para la comunicación entre las empresas, clientes y los proveedores, y el aporte de estos sistemas en la interacción óptima con clientes y proveedores.

\subsection{Encuesta de validación}

Como se mencionó en la sección de Metodología, durante la fase de validación con los stakeholders del proyecto, se aplicó un segundo instrumento que incluyó mejoras con respecto al inicial que, a pesar de ser muy completo, recibió críticas por su extensión y nivel de complejidad.

Con el fin de tener una segunda muestra de medición en las áreas de estudio, se diseñó el siguiente cuestionario (ver Tabla 1). Los resultados de este cuestionario son los que se presentarán en este trabajo.

\section{Procedimiento}

Una vez diseñado el instrumento de diagnóstico, se procedió a realizar un proceso de sondeo con los departamentos, con el fin de adquirir los datos que sirvieran para validar los indicadores definidos y tener la medición de su desempeño propio. Este documento fue enviado a los directores de tecnología informática en los diferentes entes territoriales del Diamante Caribe y Santanderes, por ser ellos las personas a cargo de los aspectos de plataformas digitales en las gobernaciones y alcaldías de las ciudades capitales.

En su primera versión, el documento se diseñó para un diligenciamiento físico; sin embargo, por dificultades en el proceso de logística de envío, como retraso en el servicio postal y tiempos de procesamiento en las oficinas, se tomó la decisión de crear una encuesta digital usando la herramienta Google Forms, con el fin de poder enviar invitaciones a través de correo electrónico a los directores.

De las invitaciones iniciales, sólo 2 de 10 ciudades diligenciaron la encuesta. De acuerdo con los comentarios de los directores entrevistados, la extensión del instrumento en medio digital, un diseño muy pobre y con pocas facilidades para diligenciarlo, generaron una resistencia inmediata de parte de los encuestados. Además, se añade el hecho de que los cinco (5) instrumentos fueron enviados simultáneamente, y cada uno con una larga extensión. Para poder obtener suficientes respuestas se tuvo que enviar personal a cada uno de los departamentos en un lapso de 3 semanas, con el fin de apoyar el proceso de diligenciamiento. Sin embargo, luego de las visitas in situ, solo la encuesta de Plataformas Tecnológicas fue la única que fue llenada por cinco (5) departamentos. El resto de las encuestas fueron diligenciadas por tres (3) o menos departamentos, e incluso hubo una que ninguno diligenció.

El único caso exitoso fue la encuesta de instrumentación industrial, la cual recibió 15 respuestas de industrias de la región. La única salvedad que hay que tomar es que existe un sesgo en los resultados, pues son empresas que ya han implementado algún tipo de automatización o que estaban en el proceso de hacerlo, por lo tanto sus resultados tienden a ser muy positivos con respecto al valor esperado de toda la industria. Por esta razón, los resultados de esa encuesta se están reservando para una investigación posterior que permita su validación y contraste con un mayor número de industrias.

En la jornada de validación con stakeholders se pudo aplicar una nueva encuesta, menos larga y más enfocada en los temas y proyectos puntuales, a un buen número de los asistentes que estaban en un espacio único, lo que permitió que se pudieran obtener muchos más resultados que en la primera versión, y recibir datos sobre todas las áreas. 
Tabla 1. Lista de preguntas para validación de los resultados del diagnóstico y los perfiles de proyectos con stakeholders

\begin{tabular}{|c|c|}
\hline Área de Estudio & Pregunta \\
\hline \multirow[t]{4}{*}{$\begin{array}{l}\text { Plataformas TI } \\
(1 . .5)\end{array}$} & $\begin{array}{l}\text { Uso de herramientas de análisis de datos (Minería de Datos, Business Intelligence, Big } \\
\text { Data, Inteligencia artificial) para la toma de decisiones. }\end{array}$ \\
\hline & $\begin{array}{l}\text { La infraestructura de cómputo con que cuento es suficiente para realizar mis tareas } \\
\text { (Servidores, redes, software especializado). }\end{array}$ \\
\hline & $\begin{array}{l}\text { Mi relación con los actores TIC (universidades, empresas, centros de investigación, } \\
\text { gobierno) son adecuadas. }\end{array}$ \\
\hline & $\begin{array}{l}\text { La infraestructura de comunicación al servicio de los ciudadanos y las empresas es } \\
\text { adecuada (cubrimiento, costos, tecnología). }\end{array}$ \\
\hline \multirow[t]{4}{*}{$\begin{array}{l}\text { E-Government } \\
(1 . .5)\end{array}$} & $\begin{array}{l}\text { La región cuenta con plataformas digitales de acceso al ciudadano que brindan } \\
\text { información sobre las actividades de gestión pública. }\end{array}$ \\
\hline & $\begin{array}{l}\text { Las plataformas digitales facilitan el acceso a información sobre las actividades de } \\
\text { gestión pública diversos temas, para divulgación pública. }\end{array}$ \\
\hline & $\begin{array}{l}\text { La región cuenta con canales de conectividad (Internet, sitios web o quioscos) que } \\
\text { permitan acceder a contenidos sobre las actividades de gestión pública. }\end{array}$ \\
\hline & La región cuenta con un sistema de apoyo para la prevención y gestión de riesgo. \\
\hline \multirow[t]{3}{*}{$\begin{array}{l}\text { Salud } \\
(1 . .5)\end{array}$} & $\begin{array}{l}\text { Disponibilidad de parte de entes gubernamentales de herramientas digitales necesarias } \\
\text { para ofrecer atención médica remota a pacientes que lo requieran. }\end{array}$ \\
\hline & $\begin{array}{l}\text { Existencia de herramientas de calidad de parte de los entes gubernamentales para } \\
\text { enfrentar problemas de salud pública. }\end{array}$ \\
\hline & $\begin{array}{l}\text { Soporte del sistema de salud con herramientas TIC para jubilados y personas de la } \\
\text { tercera edad en general, para ofrecer asistencia de manera adecuada y oportuna. }\end{array}$ \\
\hline \multirow[t]{5}{*}{$\begin{array}{l}\text { Educación } \\
(1 . .5, \text { No responde }- \text { NR) }\end{array}$} & Calidad de los equipos de cómputo de los establecimientos educativos. \\
\hline & Calidad de los recursos conectividad y comunicaciones de los planteles educativos. \\
\hline & Calidad del software disponible para el apoyo a procesos de aprendizaje. \\
\hline & Calidad del capital humano requerido para mantener iniciativas de emprendimiento. \\
\hline & $\begin{array}{l}\text { Pertinencia del sistema educativo en la formación de ciudadanos en el nuevo contexto } \\
\text { de territorio Diamante. }\end{array}$ \\
\hline \multirow[t]{3}{*}{$\begin{array}{l}\text { Instrumentación de ciudad } \\
\text { (No sabe - NS, Sí, No) }\end{array}$} & $\begin{array}{l}\text { Existe algún banco de información centralizado que contiene indicadores de gestión de } \\
\text { las ciudades. }\end{array}$ \\
\hline & $\begin{array}{l}\text { Los planes de gobierno locales se alinean al uso de información obtenida por parte de } \\
\text { las plataformas tecnológicas. }\end{array}$ \\
\hline & $\begin{array}{l}\text { Es importante la percepción y la gestión de información como herramienta clave para el } \\
\text { gobierno de las ciudades. }\end{array}$ \\
\hline
\end{tabular}

\section{Resultados}

Esta sección se divide en dos partes: resultados de la encuesta de validación con stakeholders, y la presentación de los nueve (9) proyectos; resultados que, luego del análisis, conforman la visión inicial del Diamante Digital para el territorio.

\subsection{Validación con stakeholders}

En términos de Plataformas TI, la Figura 1 muestra varias tendencias interesantes: de acuerdo con los encuestados, el uso de herramientas de análisis de datos tiene una baja percepción por parte de los actores; las valoraciones no pasan de un 3 sobre 5, teniendo la mitad entre los valores 1 y 2 . Esto significa que el uso de estas herramientas por parte de los entes territoriales no es evidente. Esto refleja un poco interés o la poca disponibilidad de datos para poder hacer análisis en pro de la planeación efectiva de ciudad. La infraestructura de cómputo tuvo mejores valoraciones, aunque la gran mayoría estuvieron en el valor de 3 , lo cual refleja que los actores no están contentos con los recursos disponibles. Las relaciones con los actores TIC tienen una amplia diversidad en sus respuestas, aunque la mayoría la colocan en un valor de 4, lo que parece indicar que, al menos para un $83 \%$ de los encuestados, las relaciones con otros actores TIC son aceptables (3). Por último, la infraestructura de comunicación con los ciudadanos y empresas está concentrada entre los valores 2 y 3 , así que el $83 \%$ de los actores la considera entre aceptable y mala. 


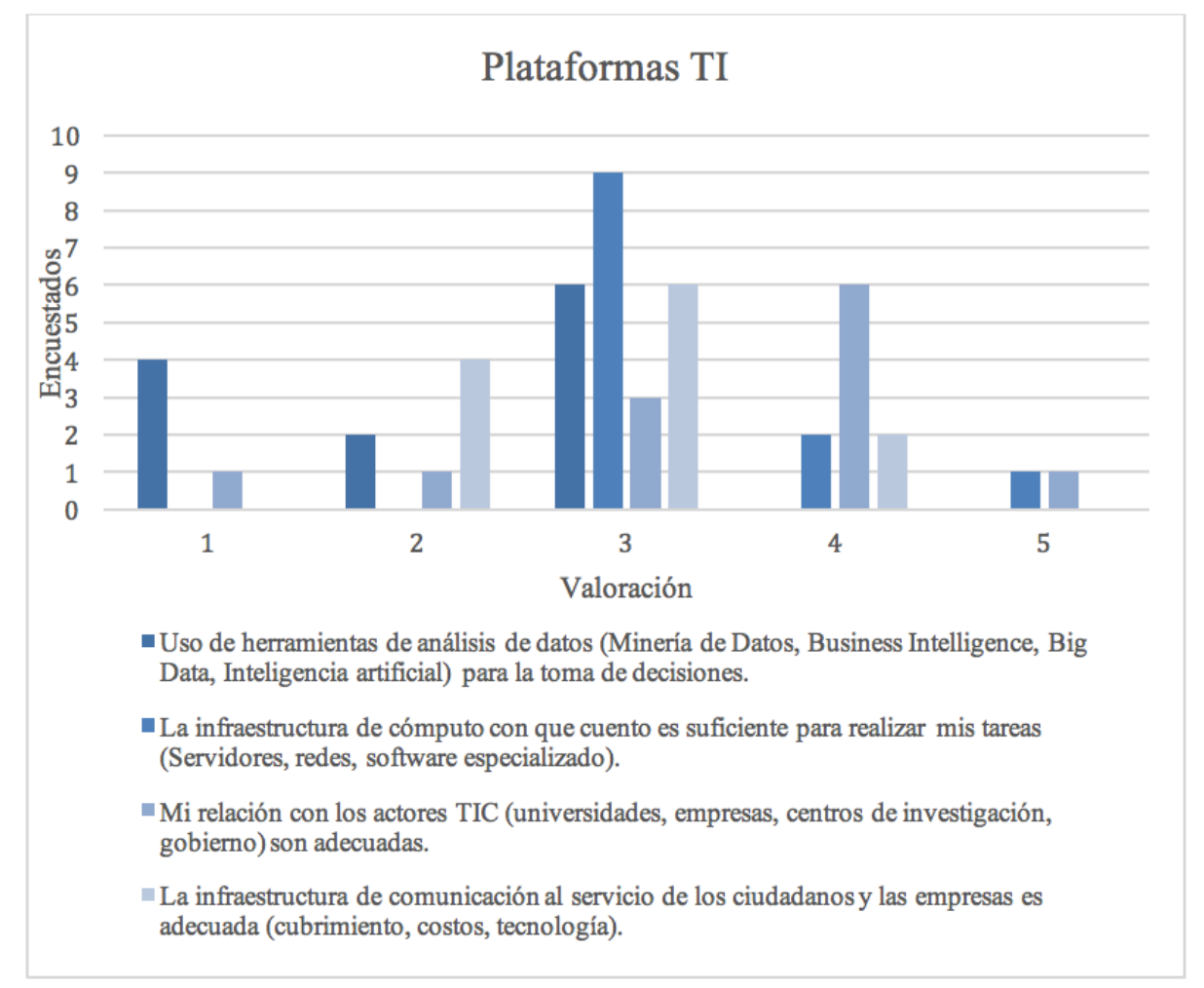

Figura 1. Resultados de la encuesta en el área de Plataformas TI

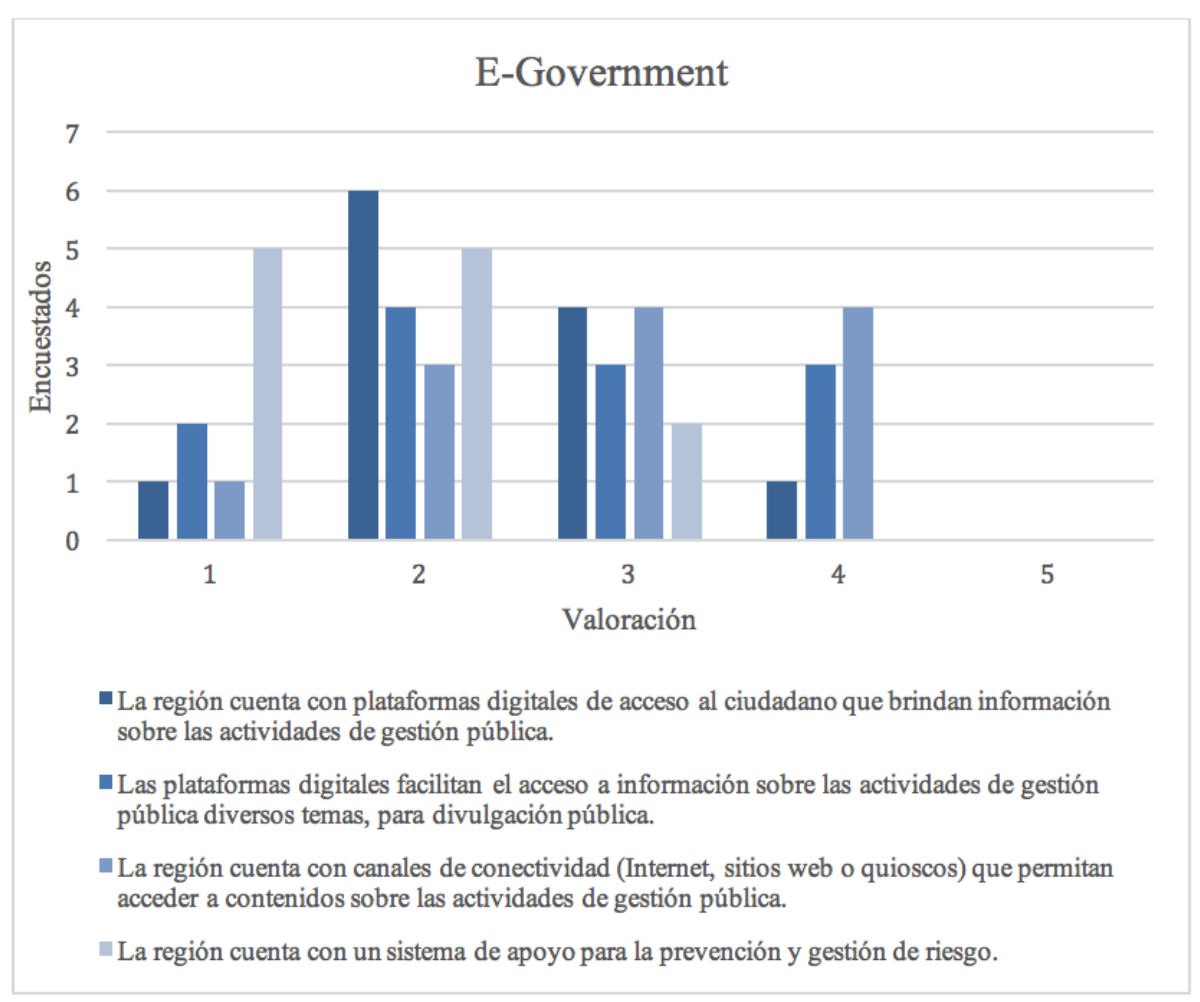

Figura 2. Resultados de la encuesta en el área de E-Government 
En lo relacionado con E-Government, la Figura 2 muestra, de forma general, cómo en ninguno de los aspectos evaluados llegó a tener una valoración de 5. El elemento que recibió mejor valoración fue la disponibilidad de canales de conectividad para acceder a contenidos de gestión pública; esto es un reflejo de la inversión realizada por el gobierno nacional y apoyada por los entes territoriales para abrir puntos y kioscos Vive Digital, y las inversiones en interconexión con fibra que se han venido haciendo en los municipios del país. Ahora, las plataformas digitales para acceder a la información tuvieron una valoración mejor distribuida y uniforme, con una gran mayoría de valoraciones entre los 2 y 3 , lo que implica una valoración. Finalmente, la falta de un sistema de apoyo para la prevención y gestión del riesgo es claramente una falencia detectada por los encuestados, en donde la gran mayoría la valoran entre 1 y 2 para la región.

En el área de salud, la Figura 3 muestra un panorama aún más complejo: las valoraciones están concentradas desde un $75 \%$ en las valoraciones de 1 y 2 , mostrando lo crítico de este sector en términos de plataformas TIC. La pregunta que tuvo mejores resultados fue la referente a las herramientas para enfrentar problemas de salud pública, que se puede explicar por la legislación que obliga el reporte a nivel nacional de información epidemiológica para su análisis y toma de decisiones en el centro del país. Por lo tanto, la atención remota de pacientes y apoyo a los jubilados y personas de la tercera edad son aspectos que requieren atención por parte de los entes territoriales.

En el área de educación, la distribución de las respuestas se concentra en un nivel aceptable, pero distribuido de manera similar entre las valoraciones 1, 2 y 4, aunque como en el caso de E-Government, la mayoría de las preguntas no alcanzó una valoración de 5; únicamente la pregunta sobre la pertinencia del sistema educativo alcanzó un 33\% de encuestados que la valoraron con el máximo valor. Las preguntas sobre infraestructura de cómputo, comunicaciones y software tuvieron un comportamiento más bien uniforme, con un poco de ventaja en la infraestructura de cómputo, que se puede explicar por los esfuerzos de programas como Computadores para Educar que han apoyado el proceso de dotación de computadores y tabletas a los colegios públicos del país (ver Figura 4).

Por último, en el área de Instrumentación de Ciudad se quiso valorar, más que la infraestructura existente en las áreas definidas en el estudio inicial, la visión de los actores en cuanto a la importancia de contar con la información que estas plataformas pueden proveer y su uso en la toma de decisiones de la ciudad.

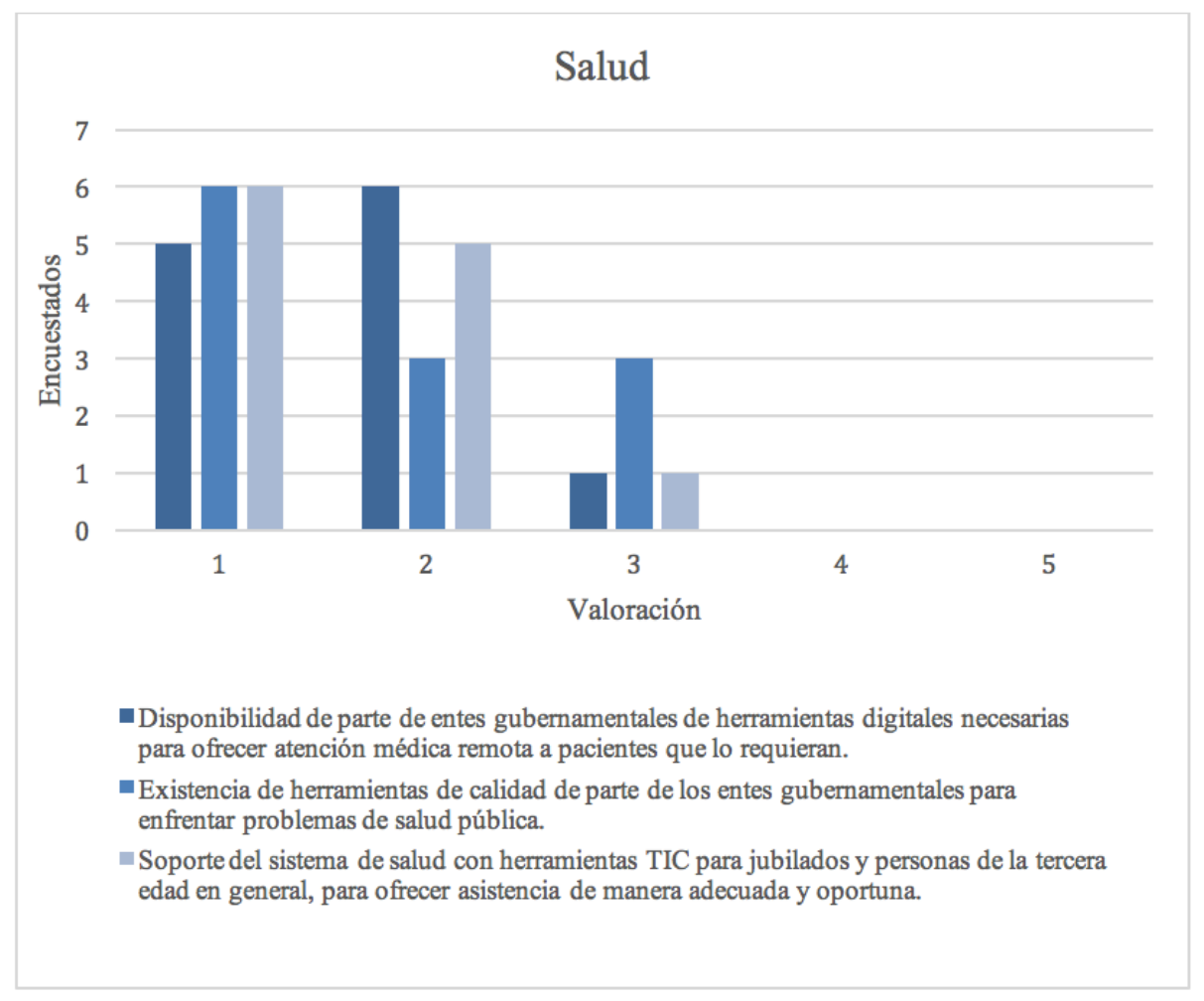

Figura 3. Resultados de la encuesta en el área de Salud 


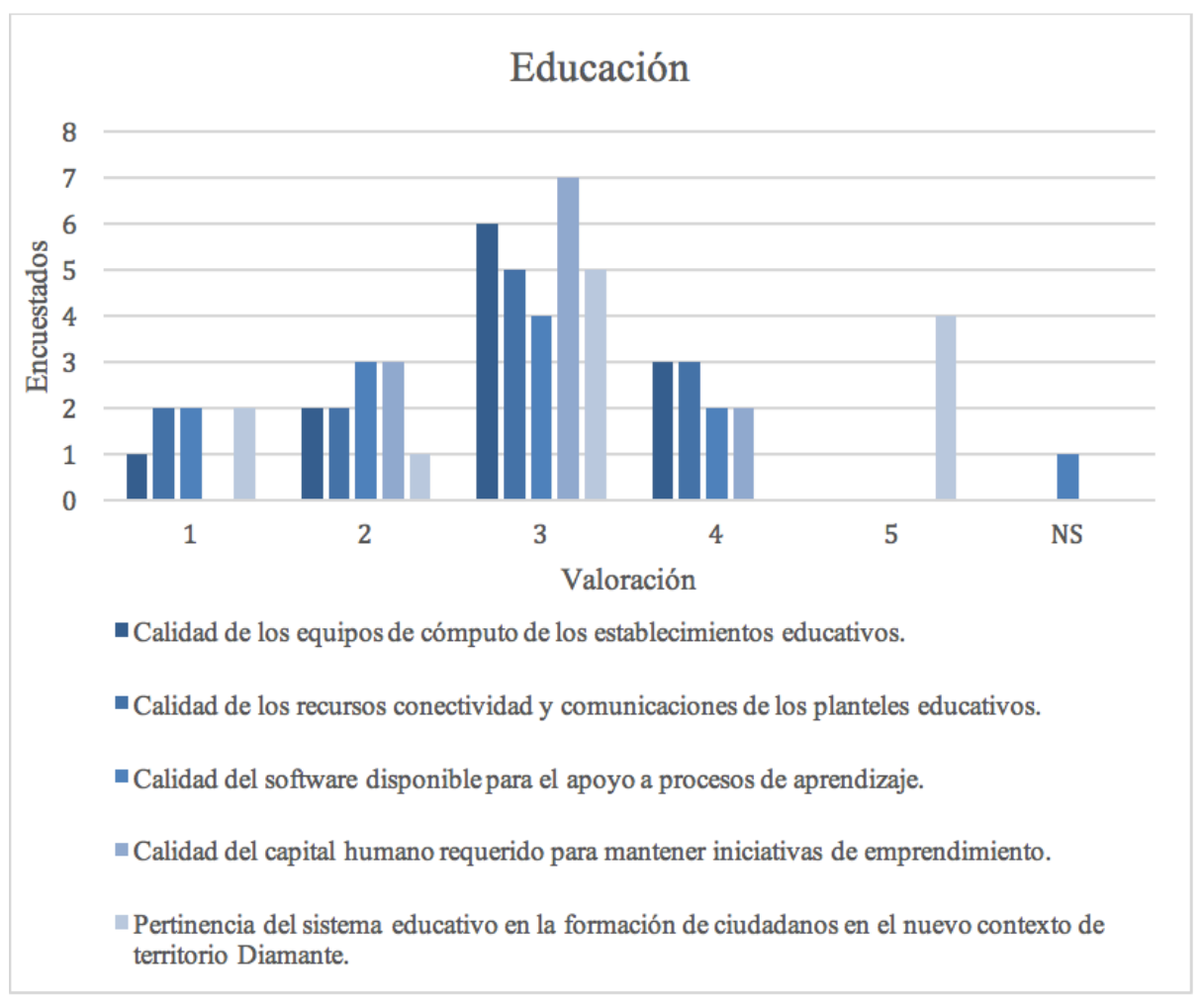

Figura 4. Resultados de la encuesta en el área de Educación

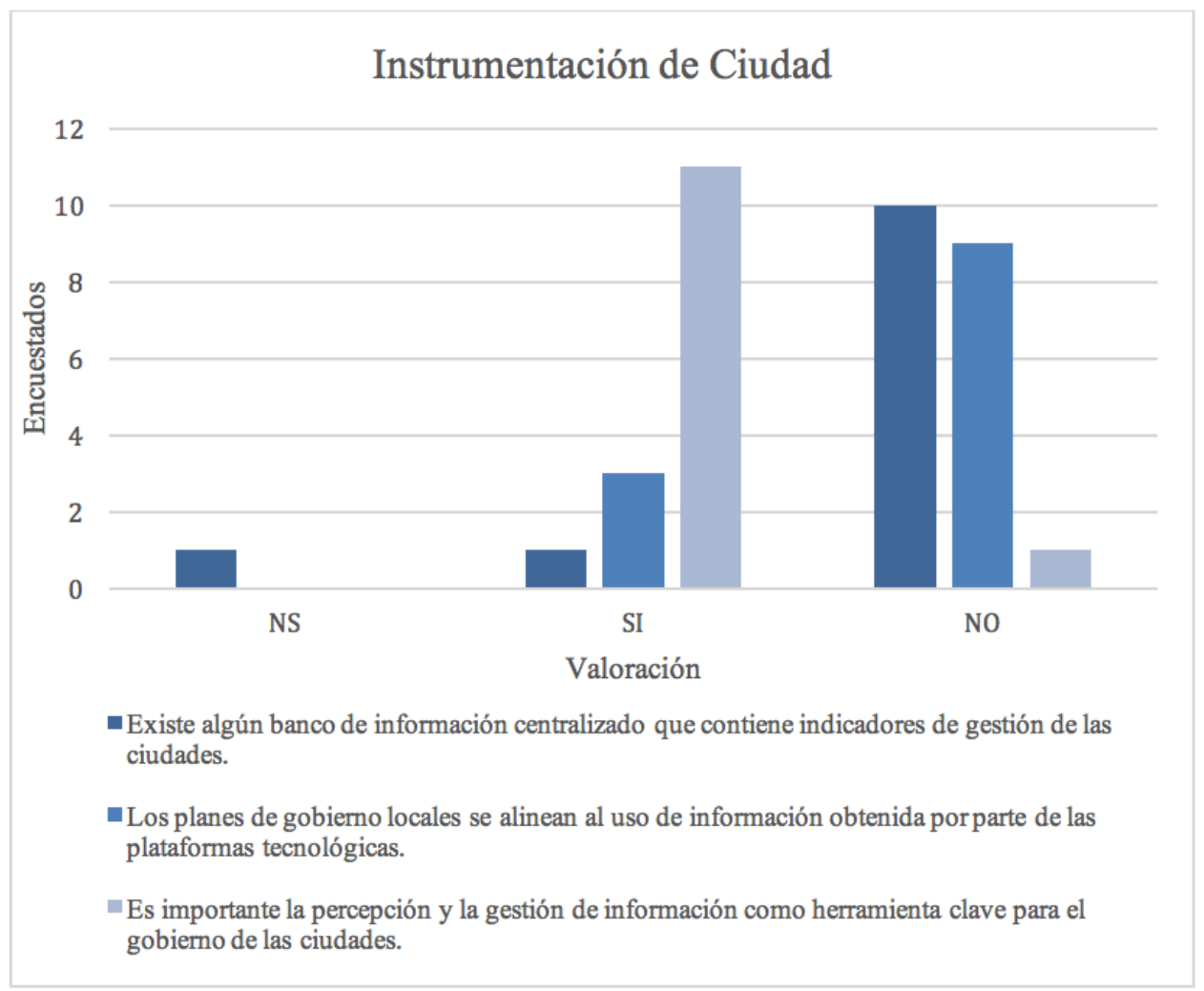

Figura 5. Resultados de la encuesta en el área de Instrumentación de Ciudad. 
La Figura 5 muestra que, en la primera pregunta sobre la existencia de indicadores de gestión para las ciudades, la gran mayoría de los actores respondieron que no existe una plataforma de apoyo. Un sistema de este estilo estaría seguramente alimentado por una infraestructura de sensores y actuadores en la ciudad para contar con información en tiempo real y poder saber el estado actual de la ciudad en cuanto a sus variables y funciones misionales.

La segunda pregunta hace referencia a si los planes de gobierno usan la información generada por las plataformas tecnológicas. Aunque las encuestas muestran que al menos un $25 \%$ de los encuestados opinan que sí existe, la gran mayoría considera que la información de las plataformas no existe o no llega a ser usada para los procesos de administración de la ciudad.

Por último, el 92\% de los actores consideran que la percepción y gestión de la información es clave para los gobiernos locales, por lo tanto, la definición de una plataforma en las grandes ciudades, en las áreas dedicadas a la agricultura y a la logística y transporte de bienes, es muy importante para el desarrollo de la región, pues del estudio inicial se pudo ver cómo estas plataformas no están bien desarrolladas en la región.

\subsection{Perfiles de proyectos}

La segunda fase de este proyecto fue la definición de un portafolio de perfiles de proyectos que fijara una infraestructura tecnológica base, no solo para homogeneizar la región con las áreas más desarrolladas del país, sino que le permitiera aumentar su competitividad y proveer un crecimiento sostenido y sostenible en el mediano y largo plazo, una vez se fueran implementando. La Tabla 2, lista los títulos y los objetivos generales de cada uno de los nueve (9) proyectos que conforman un proyecto de desarrollo regional conjunto denominado Diamante Digital.

Tabla 2. Lista de perfiles de proyectos para el proyecto Diamante Digital

\begin{tabular}{|c|c|}
\hline Título & Objetivo general \\
\hline $\begin{array}{l}\text { Sistema integrado piloto de monitoreo, } \\
\text { análisis y administración ambiental. }\end{array}$ & $\begin{array}{l}\text { Diseñar e implementar una plataforma de información en tiempo } \\
\text { real para la adquisición, análisis y presentación de variables medio } \\
\text { ambientales y manejo de alertas tempranas para la región. }\end{array}$ \\
\hline $\begin{array}{l}\text { Sistema de análisis de potencial de } \\
\text { implementación de energías renovables. }\end{array}$ & $\begin{array}{l}\text { Diseñar e implementar una plataforma de información/análisis para } \\
\text { establecer el potencial de generación eléctrica distribuida en entornos } \\
\text { rural y urbano dependientes de la ubicación geográfica. }\end{array}$ \\
\hline $\begin{array}{l}\text { Plataforma de apoyo para la toma de } \\
\text { decisiones en planificación urbana. }\end{array}$ & $\begin{array}{l}\text { Diseñar e implementar una plataforma informática integral de } \\
\text { apoyo a la gestión urbana que incluya servicios como la integración } \\
\text { de datos existentes en las fuentes gubernamentales, participación } \\
\text { ciudadana, publicación de datos abiertos al público y simulación de } \\
\text { ciudad, como apoyo a la toma de decisiones de los entes territoriales } \\
\text { del Territorio Diamante. }\end{array}$ \\
\hline $\begin{array}{l}\text { Modelo de implementación de agricultura } \\
\text { de precisión. }\end{array}$ & $\begin{array}{l}\text { Desarrollar las capacidades tecnológicas y competitivas del campo } \\
\text { en el Caribe Colombiano a través de la aplicación de la agricultura de } \\
\text { precisión y las plataformas de información geográfica. }\end{array}$ \\
\hline $\begin{array}{l}\text { Implementación de la plataforma } \\
\text { información agronómica. }\end{array}$ & $\begin{array}{l}\text { Diseñar e implementar una plataforma de información enfocada a la } \\
\text { gestión de la oferta y la demanda de productos, servicios derivados } \\
\text { de la caracterización del sector agroindustrial de la región caribe } \\
\text { colombiana y en especial de los productos con potencial exportador. }\end{array}$ \\
\hline $\begin{array}{l}\text { Plataforma de innovación abierta para } \\
\text { conectar la oferta científico-tecnológica con } \\
\text { la demanda del sector productivo. }\end{array}$ & $\begin{array}{l}\text { Fortalecer la articulación entre la oferta científico-tecnológica y la } \\
\text { demanda del sector empresarial del Territorio Diamante Caribe y } \\
\text { Santanderes. }\end{array}$ \\
\hline $\begin{array}{l}\text { Implementación de la plataforma } \\
\text { información logística. }\end{array}$ & $\begin{array}{l}\text { Diseñar e Implementar una plataforma de información enfocada a la } \\
\text { gestión de la oferta y la demanda de productos, servicios derivados de } \\
\text { la caracterización del sector logístico en la región Caribe Colombiana } \\
\text { y en especial asociados con los canales de exportación e importación. }\end{array}$ \\
\hline
\end{tabular}

Sistema integrado inteligente basado en

Big Data y soluciones tecnológicas para el Aumentar el nivel de uso y apropiación de las TIC en el sector salud sector salud del territorio Diamante Caribe y del Territorio Diamante Caribe y Santanderes.

Santanderes.

Sistema integrador inteligente para el sector Diseñar e implementar una plataforma de información inteligente que turístico. facilite la toma de decisiones en el sector turismo. 


\section{Conclusiones}

La región Caribe, de acuerdo con los resultados del diagnóstico y de los instrumentos aplicados a los actores regionales mostró un panorama complicado: una región con un nivel de desarrollo tecnológico aceptable solo en algunas de las capitales de la región, con rezagos en áreas tan importantes como la salud y la educación, y que no usa la información que tiene (si es que la tiene) para su propia planeación.

Aunque para muchos actores esto podría ser un escenario desolador y desmotivador, las conclusiones del equipo del proyecto es que este resultado revela un camino hacia el cual hay que moverse, un objetivo claro por el cual hay que trabajar de la mano con la academia, la industria y el gobierno. En la reunión con los stakeholders se pudo apreciar el interés de los tres grandes actores (academia, gobierno e industria), por trabajar para la región en los grandes proyectos de infraestructura digital que se necesitan para sentar las bases del desarrollo de la región.

La industria TIC está muy interesada en participar activamente en la implementación de estos proyectos pues, además de la posibilidad que tiene de crecer y posicionarse como un socio importante para el gobierno, es consciente que una economía más dinámica en la región es un beneficio para todos. La academia, además de querer ser el puente para la formación de más y mejor capital humano para la industria TIC y los demás sectores productivos y sector gobierno, quiere convertirse en un socio para los procesos de investigación, desarrollo e innovación que le permitirán a la industria ofrecer mejores productos y servicios acordes con las necesidades locales, y con miras a la comercialización a nivel nacional e internacional. Por último, el Gobierno nacional tiene un fuerte interés en que estas nuevas tecnologías lleguen a todos los municipios, pues reconocen el valor que pueden tener en la calidad de vida de los ciudadanos, y los entes territoriales cada vez están más abiertos a incluir estas herramientas tecnológicas para optimizar sus procesos de gestión.

Por otro lado, dentro del marco del proyecto se lograron formular y desarrollar de forma integral trabajos de tesis doctoral y de maestría en la línea de Ciudades y Territorios Inteligentes, como por ejemplo, el trabajo desarrollado por la doctora Gina Maestre Góngora, denominado "Framework de Gestión de Tecnologías de Información para Ciudades Inteligentes: Caso Colombiano", presentado en el mes de junio de 2018. El objetivo general de este trabajo fue el diseño y evaluación de un framework que permite valorar la capacidad de gestión de tecnología de información en ciudades inteligentes colombianas para orientar rutas de mejoramiento evolutivo en las estrategias y proyectos soportados en tecnologías de información. Adicionalmente, en el marco del desarrollo de trabajos de maestría, se llevaron a cabo dos proyectos: el desarrollo un modelo de madurez de capacidad para Smart Cities, el cual toma como punto de partida una revisión sistemática de la literatura enmarcada dentro de los estándares y rankings de medición a nivel mundial, que busca medir el nivel de madurez o desarrollo de las ciudades inteligentes; y la definición de un modelo de interpolación geográfica para material particulado PM10, que pretende ser aplicado en sistema de información centrado en los ciudadanos para que puedan calcular y predecir a corto plazo su exposición a contaminantes. Este tipo de iniciativas académicas, relacionadas con variables puntuales de problemas en las ciudades, no hubiera sido posible si no se contara con los estudios preliminares que fundamentaron este proceso.

Sin embargo, en el proceso también hubo dos importantes lecciones aprendidas. Durante las fases de adquisición y validación de información, el nivel de colaboración de las entidades territoriales no fue el ideal: en algunos casos se apreciaba falta de interés por parte de los funcionarios, quienes no estaban motivados adecuadamente para participar en los ejercicios a pesar de ser un lineamiento transversal a nivel de departamento y alcaldías. Además, las últimas fases del proyecto, la formulación de los proyectos del Diamante Digital, se dieron justo en medio del empalme entre las administraciones departamentales en las últimas elecciones. Por cuestiones coyunturales, estos proyectos no alcanzaron a presentarse a los gobernadores y, por lo tanto, no se incluyeron en los respectivos planes de desarrollo. Esto significó la no destinación de presupuesto para la implementación de estos proyectos $\mathrm{y}$, durante los primeros años de las administraciones actuales, no se pudo concretar ni la iniciativa, ni proyectos individuales del portafolio. Estas dos experiencias permiten concluir que futuras iniciativas similares deben estar claramente alineadas con los tiempos de construcción de los planes de gobierno, con el fin de garantizar compromiso real, incluyendo actividades y presupuesto específico en cada una de las secretarías involucradas.

El Diamante Digital se presentó como una oportunidad de pensar en las TIC no como un accesorio de alto costo, sino como una herramienta capaz de conectar actores estratégicos, conocer el territorio a través de sus datos, repensar el desarrollo urbano y rural, garantizar acceso a recursos públicos para todos los ciudadanos y lograr una gestión transparente, sostenible y participativa. El portafolio de proyectos es una herramienta aún vigente y pertinente para el escenario de la Costa Caribe y Santanderes, por lo que 
se espera sean nuevamente presentados en el corto plazo a los futuros directivos regionales, tanto en los departamentos como en la naciente RAP Caribe.

\section{Agradecimientos}

Este proyecto fue financiado por el Sistema General de Regalías con el apoyo de las gobernaciones de Atlántico, Bolívar, Cesar, Córdoba, La Guajira, Magdalena, Norte de Santander, Santander y Sucre, a través del proyecto "Análisis de los factores clave de competitividad para la construcción de un nuevo modelo de territorio inteligente en la región Caribe y Santanderes", que contó, a su vez, con la participación de Findeter, Fundación Metrópoli, Microsoft, la Universidad del Norte y la Universidad Industrial de Santander.

\section{Declaración de conflicto de intereses}

Los autores declaran no tener conflicto de intereses con respecto a la investigación, autoría y/o publicación de este artículo.

\section{Referencias}

Alawadhi, S., Aldama-Nalda, A., Chourabi, H., Gil-Garcia, J. R., Leung, S., Mellouli, S., \& Walker, S. (2012). Building Understanding of Smart City Initiatives. In H. J. Scholl, M. Janssen, M. A. Wimmer, C. E. Moe, \& L. S. Flak (Eds.) (pp. 40-53). Berlin, Heidelberg: Springer Berlin Heidelberg. https://doi. org/10.1007/978-3-642-33489-4_4

Albino, V., Berardi, U., \& Dangelico, R. M. (2015). Smart Cities: Definitions, Dimensions, Performance, and Initiatives. Journal of Urban Technology, 22(1), 3-21. https://doi.org/10.1080/10630732.2014.942092

Deloitte Research. (2000). At the dawn of e-Government: the citizen as customer / Deloitte Research. - Version details - Trove. New York: Deloitte Consulting.

Department of Economic and Social Affairs. (2018). World Urbanization Prospects: The 2018 Revision. New York.

Division for Public Institutions and Digital Government. (2018). UN E-Government Survey 2018. New York.

Dutton, W. H., Blumler, J. G., \& Kraemer, K. L. (1987). Wired cities: shaping the future of communications. Wired cities: shaping the future of communications. Washington Program, Annenberg School of Communications.

Fundación Metrópoli. (2013). Diamante Caribe y Santanderes de Colombia. Enfoque Conceptual, Metodología y Plan de Trabajo. Bogotá.

Grima-Izquierdo, C. (2010). A generic architecture for e-Government and e-Democracy: requirements, design and security risk analysis. Lap Lambert Academic Publ.

Hai, C., \& Jeong, I. (2007). Fundamental of development administration. Scholar Press.

Harrison, C., \& Donelly, A. (2011). A Theory of Smart Cities. In Proceedings of the 55th Annual Meeting of the ISSS (pp. 1-15). Hull, UK.

Hyang-Sook, C., Byung-Sun, C., \& Woong-Hee, P. (2007). Ubiquitous-City Business Strategies: The Case of South Korea. In PICMET '07 - 2007 Portland International Conference on Management of Engineering Technology (pp. 1147-1153). https://doi.org/10.1109/PICMET.2007.4349438

Ishida, T., \& Isbister, K. (2000). Digital cities: technologies, experiences, and future perspectives. Springer.

Kaylor, C., Deshazo, R., \& Van Eck, D. (2001). Gauging e-government: A report on implementing services among American cities. Government Information Quarterly, 18(4), 293-307. https://doi.org/10.1016/S0740624X(01)00089-2

Komninos, N. (2002). Intelligent cities: innovation, knowledge systems, and digital spaces. Spon Press.

Leem, Y., Lee, S. H., \& Yoon, J. (2014). Linking Data and Converging Systems for Smarter Urban Services: Two Cases of U-City Service in Korea. Procedia Environmental Sciences, 22, 89-100. https://doi.org/10.1016/j. proenv.2014.11.009 
Marsal-Llacuna, M.-L., Colomer-Llinàs, J., \& Meléndez-Frigola, J. (2015). Lessons in urban monitoring taken from sustainable and livable cities to better address the Smart Cities initiative. Technological Forecasting and Social Change, 90(90), 611-622. https://doi.org/10.1016/j.techfore.2014.01.012

Neirotti, P., De Marco, A., Corinna Cagliano, A., Mangano, G., \& Scorrano, F. (2014). Current trends in Smart City initiatives: Some stylised facts. Cities, 38, 25-36. https://doi.org/10.1016/j.cities.2013.12.010

Rogers, R. G., \& Gumuchdjian, P. (1997). Cities for a small planet. Faber and Faber.

Streitz, N. A. (2011). Smart Cities, Ambient Intelligence and Universal Access. In C. Stephanidis (Ed.), International Conference on Universal Access in Human-Computer Interaction (pp. 425-432). Berlin, Heidelberg: Springer Berlin Heidelberg. https://doi.org/10.1007/978-3-642-21666-4_47

Yin, C., Xiong, Z., Chen, H., Wang, J., Cooper, D., \& Bertrand, D. (2015). A Literature Survey On Smart Cities. Science China Information Sciences, 58(10), 1-18. https://doi.org/10.1007/s11432-015-5397-4

Yovanof, G. S., \& Hazapis, G. N. (2009). An Architectural Framework and Enabling Wireless Technologies for Digital Cities \& Intelligent Urban Environments. Wireless Personal Communications, 49(3), 445-463. https://doi.org/10.1007/s11277-009-9693-4

Zygiaris, S. (2013). Smart City Reference Model: Assisting Planners to Conceptualize the Building of Smart City Innovation Ecosystems. Journal of the Knowledge Economy, 4(2), 217-231. https://doi.org/10.1007/ s13132-012-0089-4 